\title{
Lifestyle, chronic diseases and self-rated health among Malaysian adults: results from the 2011 National Health and Morbidity Survey (NHMS)
}

Ying Ying Chan ${ }^{1 *}$, Chien Huey Teh², Kuang Kuay Lim', Kuang Hock Lim', Pei Sien Yeo ${ }^{1}$, Chee Cheong Kee ${ }^{2}$, Mohd Azahadi Omar ${ }^{1}$ and Noor Ani Ahmad ${ }^{1}$

\begin{abstract}
Background: Self-rated health (SRH) has been demonstrated as a valid and appropriate predictor of incident mortality and chronic morbidity. Associations between lifestyle, chronic diseases, and SRH have been reported by various population studies but few have included data from developing countries. The aim of this study was to determine the prevalence of poor SRH in Malaysia and its association with lifestyle factors and chronic diseases among Malaysian adults.
\end{abstract}

Methods: This study was based on 18,184 adults aged 18 and above who participated in the 2011 National Health and Morbidity Survey (NHMS). The NHMS was a cross-sectional survey (two-stage stratified sample) designed to collect health information on a nationally representative sample of the Malaysian adult population. Data were obtained via face-to-face interviews using validated questionnaires. Two categories were used to measure SRH: "good" (very good and good) and "poor" (moderate, not good and very bad). The association of lifestyle factors and chronic diseases with poor SRH was examined using univariate and multivariate logistic regression.

Results: Approximately one-fifth of the Malaysian adult population (20.1 \%) rated their health as poor (men: $18.4 \%$ and women: $21.7 \%$ ). Prevalence increases with age from $16.2 \%$ (aged 18-29) to $32.0 \%$ (aged $\geq 60$ ). In the multivariate logistic regression analysis, lifestyle factors associated with poor SRH included: underweight $(\mathrm{OR}=1.29$; $95 \% \mathrm{Cl}: 1.05-1.57)$, physical inactivity ( $\mathrm{OR}=1.25 ; 95 \% \mathrm{Cl}: 1.11-1.39)$, former smoker (OR=1.38; $95 \% \mathrm{Cl}: 1.12-1.70)$, former drinker ( $\mathrm{OR}=1.27 ; 95 \% \mathrm{Cl}: 1.01-1.62)$, and current drinker $(\mathrm{OR}=1.35 ; 95 \% \mathrm{Cl}$ : 1.08-1.68). Chronic diseases associated with poor SRH included: asthma (OR $=1.66 ; 95 \% \mathrm{Cl}: 1.36-2.03)$, arthritis (OR=1.87; $95 \%$ Cl: 1.52-2.29), hypertension ( $\mathrm{OR}=1.39 ; 95 \% \mathrm{Cl}: 1.18-1.64)$, hypercholesterolemia $(\mathrm{OR}=1.43 ; 95 \% \mathrm{Cl}: 1.18-1.74)$, and heart disease $(\mathrm{OR}=1.85 ; 95 \% \mathrm{Cl}: 1.43-2.39)$.

Conclusions: This study indicates that several unhealthy lifestyle behaviours and chronic diseases are significantly associated with poor SRH among Malaysian adults. Effective public health strategies are needed to promote healthy lifestyles, and disease prevention interventions should be enhanced at the community level to improve overall health.

Keywords: Self-rated health, lifestyle, chronic diseases, National Health and Morbidity Survey, Malaysia

\footnotetext{
* Correspondence: chan.yy@moh.gov.my

${ }^{1}$ Institute for Public Health, National Institutes of Health, Ministry of Health

Malaysia, Kuala Lumpur, Malaysia

Full list of author information is available at the end of the article
} reproduction in any medium, provided you give appropriate credit to the original author(s) and the source, provide a link to the Creative Commons license, and indicate if changes were made. The Creative Commons Public Domain Dedication waiver (http://creativecommons.org/publicdomain/zero/1.0/) applies to the data made available in this article, unless otherwise stated. 


\section{Background}

A variety of lifestyle factors and health-related behaviours such as smoking, alcohol consumption, physical activity, and dietary habits can affect a person's health. An unhealthy lifestyle often results in a higher risk of chronic diseases such as heart disease, hypertension, hypercholesterolemia, and diabetes [1, 2]. Previous empirical studies have found that lifestyle factors and chronic diseases are strongly associated with self-rated health (SRH) [3-5]. Several socio-environmental determinants such as infrastructure, housing conditions, political situation, access to health care services, availability of clean water, and affordability of healthy foods are also found to be closely related to SRH [6-8]. SRH is an established predictor of morbidity and mortality and has been widely used to measure health inequalities in public health research $[9,10]$. It has been recognised as a reliable and valid health indicator that is based on a simple question in which respondents are asked to rate their current general health status on a four- or five-point scale ranging from "very good (excellent)" to "very poor" [9]. The SRH is frequently used in population health surveys because the data are easily collected; SRH has been extensively studied in Western populations [11].

Understanding the correlates of SRH can help public health professionals prioritise health-promotion and disease-prevention interventions. To date, studies that have investigated the determinants of SRH have focused primarily on Western countries, although we have found a amall number of studies that examine the topic from the perspective of developing countries in the Asian regions. Previous studies from Singapore [12], Pakistan [13], and Japan [14] have found that physical activity, tobacco use, and BMI have shown a significant association with self-reported fair or poor health. Another study from Thailand reported that chronic diseases, functional status, and psychosocial symptoms were the strongest determinants of poor SRH among Thai elderly [15]. The increasing research interest in SRH in Asian developing countries warrants this study of SRH in Malaysia, not only to provide data on SRH comparable to that of neighbouring countries such as Thailand and Singapore, but also to provide evidence-based results for developing appropriate public health policies and programmes that improve the overall health of the population.

In Malaysia, few studies have examined the topic of SRH. A recent study by Cheah [16] reported that sociodemographic characteristics, lifestyle factors, and chronic diseases have a substantial impact on SRH. However, the data were from only one state (Penang) and may not be representative of the country as a whole. Other studies focus only on exploring the socio-demographic determinants of SRH among the elderly $[17,18]$. As such, the information gathered from these studies is not sufficient for policy implementation at the national level. Hence, the present study aims to fill the gaps and improve the existing knowledge on determinants of SRH among the general population in Malaysia. Using data from the $2011 \mathrm{Na}$ tional Health and Morbidity Survey (NHMS), we determined the prevalence of poor SRH and its possible determinants-with an emphasis on relationships between lifestyle factors, chronic diseases, and SRH-in a large sample of the Malaysian adult population.

\section{Methods}

\section{Study design and data collection}

The NHMS 2011 was a nationwide, cross-sectional, population-based survey that used a two-stage stratified sampling design to select a nationally representative sample. The Malaysian states constituted the primary strata, with further stratification by urban-rural residence. The primary sampling units were enumeration blocks (EBs) provided by the Malaysian Department of Statistics from the 2010 census. Living quarters (LQs) represent the secondary sampling units. A total of 794 EBs (484 urban and 310 rural) were systematically selected from all the Malaysian EBs (about 75,000) via a probability-proportional-tosize sampling technique. Subsequently, 12 LQs were randomly selected from the approximately 80 to 120 LQs in each EB. Finally, all households and eligible household members in the selected LQs were included in the sample. The survey methodology is described in greater detail by Fadhli et al. [19].

Data collection for the NHMS 2011 was carried out from April to July 2011. Information on SRH, sociodemographic characteristics, lifestyle, and self-reported medically-diagnosed chronic diseases was obtained by trained interviewers via face-to-face interviews. The interviewers were extensively trained and conducted mock interviews prior to actual data collection. The interviewing process was closely monitored by field supervisors, who were health care personnel. A total of 18,231 eligible adults aged 18 years and older, living in the sampled households, were interviewed. Of these, 18,184 responded to the SRH questionnaire section of the NHMS 2011, giving a response rate of $99.7 \%$. To ensure a high response rate, unoccupied households were revisited up to three times. All of the eligible respondents provided written consent to be interviewed for the survey. The study protocol was approved by the Malaysian Medical and Research Ethics Committee (MREC), Ministry of Health Malaysia (NMRR-10-757-6837).

\section{Measures}

\section{Dependent variable}

Self-rated health was measured based on the question: "In general, how would you rate your health today?" There were five response categories: "very good", "good", 
"moderate", "not good" and "very bad". For purposes of analysis, the response categories were combined into two groups: "Good", which included "very good" and "good", and "Poor" which included "moderate", "not good" and "very bad". "Good" SRH served as the reference category in the logistic regression analyses.

\section{Independent variables}

Socio-demographic variables included sex, age, ethnicity, residential area (urban/rural), marital status (single, married, widow/widower/divorcee), educational level (no formal education, primary, secondary, tertiary), current work status (working/not working) and monthly household income. Age was categorised into five groups: 1829 years, 30-39 years, 40-49 years, 50-59 years, 60 years and above. Ethnicity was categorised into five groups: Malays, Chinese, Indians, other Bumiputeras (including indigenous Orang Asli in Peninsular Malaysia and the indigenous groups in Sabah and Sarawak such as Kadazan, Murut, Bajau, Melanau, Iban and Bidayuh), and "Others" (including small minority groups that settled in Malaysia such as Sikh, Serani, Portuguese, Indonesian, and Bangladeshi). Monthly household income was grouped into four levels: less than MYR1000, MYR1000-MYR1999, MYR2000-MYR2999, MYR3000 and above [1 Malaysian Ringgit $(M Y R) \approx 0.29$ US Dollar (USD)].

Lifestyle variables included body mass index (BMI), consumption of fruits and vegetables, physical activity, smoking, and alcohol consumption. Respondents' weight and height were measured using the Tanita 318 digital weighing scale (Tanita Corp., Tokyo, Japan) and the Seca 206 Bodymeter measuring tape (seca GmbH \& Co. KG, Hamburg, Germany), to the nearest $0.1 \mathrm{~kg}$ and $0.1 \mathrm{~cm}$, respectively. BMI was calculated as weight divided by height in meters squared $\left(\mathrm{kg} / \mathrm{m}^{2}\right)$. According to the World Health Organization (WHO 2000) guidelines [20], BMI was categorised according to four levels of nutritional status for both men and women: underweight $(<18.5)$, normal (18.5-24.9), overweight (25.0-29.9) and obese $(\geq 30)$.

Consumption of fruits and vegetables was measured based on four questions: i) "In a typical week, how many days do you eat fruits?" ii) "Usually on the day you eat fruits, how many servings of fruits did you eat in a day?" iii) "In a typical week, how many days do you eat vegetables", and iv) "Usually on the day you eat vegetables, how many servings of vegetables did you eat in a day?" Photographs of foods were used to help respondents recall the serving sizes of the fruits and vegetables they had consumed. The photographs showed single servings of commonly consumed fruits and vegetables such as the following: one medium-sized apple, one mediumsized banana, one slice of papaya, one slice of watermelon, eight medium-sized grapes, one cup of chopped raw leafy green vegetables or ulam (Malaysian salad herbs), and a half cup of cooked chopped eggplant or tomato or carrot or leafy green vegetables. According to the WHO STEPS criteria [21], responses to these four questions were used to obtain an assessment of total average consumption of fruits and vegetables per day, without differentiating between the two categories. Based on the WHO recommendations [22], consumption of fruits and vegetables was divided into two groups: adequate consumption of fruits and vegetables ( $\geq 5$ servings per day), and inadequate consumption of fruits and vegetables ( $<5$ servings per day).

The level of physical activity was determined based on the short form of the International Physical Activity Questionnaire (IPAQ), the reliability and validity of which have been established [23]. The original English version of the questionnaire was translated into Bahasa Malaysia, then back-translated into English for verification. The Malay version of IPAQ was pilot tested on 32 adults for concurrent validity (correlation with the original English version) by comparing the correlation of total physical activity from the Malay version with the one obtained from the original English version. An interval of one hour was allowed between administration of the two questionnaires and a good concurrent validity with Spearman correlation coefficient $(\rho)$ of 0.995 for total physical activity was obtained. The IPAQ short form was designed to estimate the overall physical activity level by assessing three specific types of physical activity (walking, moderate-intensity activities and vigorous-intensity activities) undertaken across a comprehensive set of domains (work-related, transportation, leisure time, and domestic/ gardening). The level of physical activity is calculated in terms of metabolic energy expended (MET minutes, or METs), based on the IPAQ analysis protocol [24]. The total physical activity score is the sum of all METs per week from walking to moderate-intensity activities to vigorous-intensity activities. Survey participants who performed any combination of walking, moderate-intensity activities or vigorous-intensity activities in the past 7 days, with a total physical activity score of at least 600 METs per week were categorised as "active". Those who achieved less than 600 METs per week were classified as "inactive".

Smoking status was divided into three categories: "never smoker" (never smoked in lifetime), "former smoker" (smoked daily or occasionally in the past but not a smoker at the time of the survey), and "current smoker" (smoked any tobacco products either daily or occasionally at the time of the survey). Alcohol consumption status was divided into three categories: "non-drinker" (no alcohol consumption for past 12 months, includes never drinkers), "former drinker" (consumed alcohol in the past but had stopped drinking for the past 12 months) and "current drinker" (consumed alcohol in the past 12 months). 
For chronic disease status, presence or absence of chronic diseases was based on self-reported information on chronic conditions diagnosed by a doctor or health care professional. Respondents were asked: "Have you ever been told by a doctor or Medical Assistant that you have __ ? followed by a list of chronic diseases included asthma, arthritis, diabetes mellitus, hypertension, hypercholesterolemia, and heart disease, to which the answer is "yes" or "no".

\section{Statistical analyses}

Frequency distributions of socio-demographic, lifestyle, and chronic disease variables were analysed by self-rated health status. Descriptive statistics were used to calculate the overall prevalence of poor SRH among Malaysian adults and the prevalence of poor SRH by sociodemographic, lifestyle and chronic disease variables. Associations of the measured factors with poor SRH were tested using chi-square tests. Univariate and multivariate logistic regression analyses were used to investigate the association between poor SRH, socio-demographic, lifestyle, and chronic disease variables, with "Good" SRH as the reference category. The results are presented as crude and adjusted odds ratios (ORs) with $95 \%$ confidence intervals (CIs). Variables that were associated with poor $\mathrm{SRH}$ at $p=<0.25$ in the univariate analysis were retained in the multivariate logistic regression model [25]. Those variables (i.e., "Others" ethnic group, overweight BMI group, and current drinkers) that by themselves were not significantly related to poor SRH in the univariate analysis, but were considered to make an important contribution in the presence of other variables, were also included in the final model. The multivariate logistic regression model was adjusted for all potential confounding factors and $p=<0.05$ was considered to be statistically significant. The statistical analyses were performed using SPSS for Windows (version 20.0) statistical software (IBM Corp., Armonk, NY, USA). All analyses were conducted using a complex sampling design to ensure that sample weights and study design were taken into consideration.

\section{Results}

Table 1 shows the distribution of socio-demographic characteristics, lifestyle factors, and chronic disease variables across the two categories of SRH ("good" and "poor") in the study sample $(n=18,184)$. The sample included more women (53.4\%) than men (46.6\%). Among the 18,184 respondents, the distribution of self-rated health was as follow: "very good" 3,468 (19.4 \%); "good" 11,031 (60.4 \%); "moderate" 3,349 (18.2\%); "not good" 316 (1.8\%); and "very bad" 20 (0.1\%) (see Additional file 1: Table S1). We combined the five categories into two, "good" ("very good" and "good") and "poor" ("moderate", "not good," and "very bad") SRH, with $79.9 \%$ ( $n=$ $14,499)$ of participants reporting good SRH, and $20.1 \%$ $(n=3685)$ reporting poor SRH. A higher proportion of women $(57.5 \%)$ than men $(42.5 \%)$ reported poor SRH. Similarly, a higher proportion of older adults (aged 60 years and above) reported poor SRH than younger adults (Table 1).

Table 2 shows the prevalence of poor SRH by sociodemographic characteristics, lifestyle factors, and chronic disease variables. Overall, the prevalence of poor SRH was higher among women $(21.7 \%)$ than men (18.4\%). The prevalence of poor SRH increased with age and was highest in the oldest age group $\geq 60$ years $(32.0 \%)$. A higher prevalence of poor SRH was also found among Other Bumiputeras, rural residents, widows/widowers/divorcees, individuals with no formal education, and those who were currently not working. Prevalence of poor SRH was significantly related to sex, age, ethnicity, residential area, marital status, education level, current work status, BMI status, physical activity status, smoking status, and chronic disease status $(p=<0.001)$. Regarding lifestyle variables, individuals who were underweight, physically inactive, and former smokers had higher prevalence of poor SRH. Survey participants who had chronic diseases such as asthma, arthritis, diabetes mellitus, hypertension, hypercholesterolemia, and heart disease were found to have a higher prevalence of poor SRH, compared with their counterparts who did not have these diseases.

Table 3 shows the results of univariate and multivariate logistic regression analyses for poor SRH by sociodemographic characteristics, lifestyle factors, and chronic disease variables. In the univariate analysis, all variables were associated with poor SRH at $p=<0.25$ except for "Others" ethnic group, monthly household income, overweight BMI group, and current drinkers. In the final multivariate logistic regression model, we included all variables that were statistically significant in univariate analysis $(p=<0.25)$ except for the "monthly household income" variable. After adjusting for all selected factors in the multivariate analysis, the odds of poor SRH were significantly higher for women compared with men, among older people (aged $\geq 60$ years) compared with younger people (aged 18-29 years), and among people living in rural areas compared with urban areas. Malays, other Bumiputeras and "Others" ethnic groups had higher odds of poor SRH compared with the Chinese. Respondents who were underweight $(p=<0.05)$, physically inactive $(p=<0.001)$, former smokers $(p=<0.01)$, current drinkers $(p=<0.01)$, and former drinkers $(p=<0.05)$ reported significantly higher odds of poor SRH compared with their respective counterparts. Self-reported medicallydiagnosed chronic diseases that were significantly associated with poor SRH were asthma, arthritis, hypertension, hypercholesterolemia, and heart disease $(p=<0.001$ for 
Table 1 Distribution of socio-demographic characteristics, lifestyle factors, and self-reported medically-diagnosed chronic diseases, by self-rated health status, NHMS 2011

\begin{tabular}{llll}
\hline Variables & $\begin{array}{l}\text { Total } \\
(\mathrm{n}=18184)\end{array}$ & \multicolumn{2}{l}{$\begin{array}{l}\text { Self-rated } \\
\text { health status }\end{array}$} \\
\cline { 3 - 4 } & & $\begin{array}{l}\text { Good } \\
(\mathrm{n}=14499)\end{array}$ & $\begin{array}{l}\text { Poor } \\
(\mathrm{n}=3685)\end{array}$ \\
& $\mathrm{n}(\%)$ & $\mathrm{n}(\%)$ & $\mathrm{n}(\%)$
\end{tabular}

Sex
Men
Women
Age group (years)
18-29
$30-39$
$40-49$
$50-59$
$\geq 60$

Ethnicity

Malays
Chinese
Indians
Other Bumiputeras

Others

Residential area

Urban
Rural
Marital status ${ }^{\mathrm{a}}$
Single
Married
Widow/widower/divorcee
Education level ${ }^{\mathrm{a}}$
No formal education
Primary
Secondary
Tertiary
Currently working ${ }^{\mathrm{a}}$
Yes
No

Monthly household income (1 MYR $\approx 0.29$ USD)

$\begin{array}{llll}<\text { RM1000 } & 3458(17.1) & 2712(16.9) & 746(18.0) \\ \text { RM1000-RM1999 } & 3669(18.2) & 2932(18.0) & 737(18.8) \\ \text { RM2000-RM2999 } & 3084(16.8) & 2463(16.9) & 621(16.6) \\ \geq \text { RM3000 } & 7973(47.9) & 6392(48.1) & 1581(46.6) \\ \text { BMI status }\left(\mathrm{kg} / \mathrm{m}^{2}\right)^{\mathrm{a}} & & & \\ \text { Underweight }(<18.5) & 1211(7.8) & 954(7.5) & 257(8.7) \\ \text { Normal (18.5-24.9) } & 7189(45.5) & 5868(46.1) & 1321(42.9) \\ \text { Overweight (25.0-29.9) } & 4842(30.6) & 3874(30.7) & 968(30.0)\end{array}$

Table 1 Distribution of socio-demographic characteristics, lifestyle factors, and self-reported medically-diagnosed chronic diseases, by self-rated health status, NHMS 2011 (Continued)

\begin{tabular}{|c|c|c|c|}
\hline Obese $(\geq 30)$ & $2593(16.2)$ & $1971(15.7)$ & $622(18.3)$ \\
\hline \multicolumn{4}{|l|}{$\begin{array}{l}\text { Consumption of fruits } \\
\text { and vegetables }{ }^{\mathrm{a}}\end{array}$} \\
\hline$<5$ servings/day & $16,844(92.8)$ & $13,402(92.6)$ & $3442(93.4)$ \\
\hline$\geq 5$ servings/day & $1314(7.2)$ & $1075(7.4)$ & $239(6.6)$ \\
\hline \multicolumn{4}{|l|}{$\begin{array}{l}\text { Physical activity } \\
\text { status }^{\mathrm{a}}\end{array}$} \\
\hline Active & $11,570(64.0)$ & $9472(65.9)$ & $2098(56.7)$ \\
\hline Inactive & $6476(36.0)$ & $4914(34.1)$ & $1562(43.4)$ \\
\hline \multicolumn{4}{|l|}{ Smoking status ${ }^{a}$} \\
\hline Never smoker & $12,769(70.8)$ & $10,199(70.9)$ & $2570(70.2)$ \\
\hline Former smoker & $1215(7.1)$ & $864(6.4)$ & $351(9.8)$ \\
\hline Current smoker & $4069(22.1)$ & $3334(22.7)$ & $735(20.0)$ \\
\hline \multicolumn{4}{|l|}{ Drinking status ${ }^{a}$} \\
\hline Non-drinker & $15,482(84.5)$ & $12,374(84.6)$ & $3108(84.0)$ \\
\hline Former drinker & $959(5.6)$ & $723(5.4)$ & $236(6.2)$ \\
\hline Current drinker & $1642(9.9)$ & $1325(10.0)$ & $317(9.9)$ \\
\hline \multicolumn{4}{|c|}{ Presence of chronic disease ${ }^{b}$} \\
\hline Asthma & $1142(6.3)$ & $781(5.5)$ & $361(9.9)$ \\
\hline Arthritis & $985(5.5)$ & $577(4.2)$ & $408(10.3)$ \\
\hline Diabetes mellitus & $1546(9.3)$ & $1082(8.5)$ & $464(12.3)$ \\
\hline Hypertension & 2679 (15.6) & $1820(13.6)$ & $859(23.6)$ \\
\hline Hypercholesterolemia & $1578(9.4)$ & $1058(8.0)$ & $520(14.5)$ \\
\hline Heart disease & $612(3.1)$ & $356(2.3)$ & $256(6.4)$ \\
\hline
\end{tabular}

each). Individuals with diabetes mellitus were not significantly associated with poor SRH in our study.

\section{Discussion}

The prevalence of poor SRH in Malaysia (20.1 \%) is similar to that reported in neighbouring Singapore (23.2 \%) [12], which may be related to similarity of both ethnic composition and socio-economic characteristics. However, the level of poor SRH is much higher in Malaysia than in Japan (9.8 \%) [26], Canada (11.9\%) [27] or the United States (16.4\%) [28]. Conversely, poor SRH is much lower in Malaysia than in Pakistan (65.1 \%) [13]. Such comparisons across countries should be viewed with caution because of differences in the characteristics of the study populations (e.g., age range, gender, ethnic groups) and differences in the methodology used (e.g., type of rating scale, method used to elicit responses, and wording of questions) [29]. Additionally, differences in health status can be linked to different geographical, cultural, socio-economic, and socio-political 
Table 2 Prevalence of poor self-rated health by socio-demographic characteristics, lifestyle factors, and chronic disease status among Malaysian adults aged 18 years and above, NHMS 2011

\begin{tabular}{llll}
\hline Variables & Prevalence (\%) & $95 \% \mathrm{Cl}$ & -value \\
\hline SOCIO-DEMOGRAPHIC & & \\
CHARACTERISTICS & &
\end{tabular}

Sex

$$
\text { Men }
$$

18.4

21.7

Total

20.1

Age group (years)

$18-29$
$30-39$
$40-49$
$50-59$
$\geq 60$

Ethnicity

Malays
Chinese
Indians
Other Bumiputeras
Others

Residential area

$\begin{array}{ll}\text { Urban } & 18.4 \\ \text { Rural } & 22.6\end{array}$

Marital status

Single
Married
Widow/widower/divorcee

15.0

21.2

28.6

Educational level

\section{No formal education}

Primary education

Secondary education

Tertiary education

Current work status

$$
\text { Working }
$$

Non-working

Monthly household income (1 MYR $\approx 0.29$ USD)

$<M Y R 1000$
MYR1000-MYR1999
MYR2000-MYR2999
$\geq$ MYR3000

LIFESTYLE FACTORS

BMI status $\left(\mathrm{kg} / \mathrm{m}^{2}\right)$
Table 2 Prevalence of poor self-rated health by socio-demographic characteristics, lifestyle factors, and

\begin{tabular}{|c|c|c|c|}
\hline Underweight $(<18.5)$ & 22.5 & $19.4-25.8$ & \\
\hline Normal (18.5-24.9) & 18.8 & $17.6-20.1$ & \\
\hline Overweight (25.0-29.9) & 19.6 & $18.0-21.2$ & \\
\hline Obese $(\geq 30)$ & 22.4 & $20.4-24.6$ & \\
\hline $\begin{array}{l}\text { Consumption of fruits and } \\
\text { vegetables }\end{array}$ & & & 0.235 \\
\hline$<5$ servings/day & 20.3 & $19.3-21.3$ & \\
\hline$\geq 5$ servings/day & 18.4 & $15.6-21.5$ & \\
\hline Physical activity status & & & $<0.001$ \\
\hline Active & 17.8 & $16.7-18.9$ & \\
\hline Inactive & 24.3 & $22.8-25.8$ & \\
\hline Smoking status & & & $<0.001$ \\
\hline Never smoker & 20.0 & $18.9-21.0$ & \\
\hline Former smoker & 27.9 & $24.8-31.2$ & \\
\hline Current smoker & 18.1 & $16.5-19.8$ & \\
\hline Drinking status & & & 0.474 \\
\hline Non-drinker & 20.0 & $19.0-21.0$ & \\
\hline Former drinker & 22.3 & $18.8-26.2$ & \\
\hline Current drinker & 19.9 & $17.2-22.9$ & \\
\hline \multicolumn{4}{|l|}{ CHRONIC DISEASE STATUS } \\
\hline Asthma & & & $<0.001$ \\
\hline Yes & 31.3 & $27.9-34.8$ & \\
\hline No & 19.4 & $18.4-20.3$ & \\
\hline Arthritis & & & $<0.001$ \\
\hline Yes & 38.0 & $34.0-42.2$ & \\
\hline No & 19.1 & $18.2-20.0$ & \\
\hline Diabetes mellitus & & & $<0.001$ \\
\hline Yes & 27.4 & $24.4-30.5$ & \\
\hline No & 19.9 & $18.9-21.0$ & \\
\hline Hypertension & & & $<0.001$ \\
\hline Yes & 31.2 & $28.9-33.5$ & \\
\hline No & 19.5 & $17.7-19.7$ & \\
\hline Hypercholesterolemia & & & $<0.001$ \\
\hline Yes & 32.0 & $29.0-35.3$ & \\
\hline No & 19.5 & $18.5-20.5$ & \\
\hline Heart disease & & & $<0.001$ \\
\hline Yes & 41.5 & $36.5-46.6$ & \\
\hline No & 19.4 & $18.5-20.4$ & \\
\hline
\end{tabular}
chronic disease status among Malaysian adults aged 18 years and above, NHMS 2011 (Continued)

factors, as well as other factors that affect perception of one's self-rated health [30,31].

$<0.001$ The present study found a significant association between specific lifestyle factors and SRH. Individuals who are underweight, physically inactive, former smokers, 
Table 3 Univariate and multivariate logistic regression analyses for poor self-rated health, Malaysian adults aged 18 years and older, by socio-demographic characteristics, lifestyle factors, and chronic disease status

\begin{tabular}{|c|c|c|c|c|}
\hline Variables & Crude OR $(95 \% \mathrm{Cl})$ & $p$-value & Adjusted $\mathrm{OR}^{\mathrm{a}}(95 \% \mathrm{Cl})$ & $p$-value \\
\hline \multicolumn{5}{|c|}{ SOCIO-DEMOGRAPHIC CHARACTERISTICS } \\
\hline \multicolumn{5}{|l|}{ Sex } \\
\hline Men & 1.00 & & 1.00 & \\
\hline Women & $1.23(1.12-1.35)$ & $<0.001$ & $1.32(1.15-1.52)$ & $<0.001$ \\
\hline \multicolumn{5}{|l|}{ Age group (years) } \\
\hline $18-29$ & 1.00 & & 1.00 & \\
\hline $30-39$ & $1.15(0.98-1.35)$ & 0.098 & $1.08(0.89-1.32)$ & 0.436 \\
\hline $40-49$ & $1.32(1.13-1.54)$ & 0.001 & $1.16(0.95-1.43)$ & 0.154 \\
\hline $50-59$ & $1.36(1.16-1.58)$ & $<0.001$ & $1.05(0.85-1.30)$ & 0.653 \\
\hline$\geq 60$ & $2.44(2.09-2.86)$ & $<0.001$ & $1.51(1.17-1.93)$ & 0.001 \\
\hline \multicolumn{5}{|l|}{ Ethnicity } \\
\hline Malays & $1.35(1.17-1.54)$ & $<0.001$ & $1.52(1.27-1.81)$ & $<0.001$ \\
\hline Chinese & 1.00 & & 1.00 & \\
\hline Indians & $1.16(0.91-1.48)$ & 0.226 & $1.20(0.92-1.57)$ & 0.187 \\
\hline Other Bumiputeras & $1.55(1.25-1.93)$ & $<0.001$ & $1.62(1.28-2.07)$ & $<0.001$ \\
\hline Others & $1.17(0.88-1.54)$ & 0.280 & $1.46(1.07-2.00)$ & 0.018 \\
\hline \multicolumn{5}{|l|}{ Residential area } \\
\hline Urban & 1.00 & & 1.00 & \\
\hline Rural & $1.29(1.15-1.45)$ & $<0.001$ & $1.15(1.01-1.31)$ & 0.032 \\
\hline \multicolumn{5}{|l|}{ Marital status } \\
\hline Single & 1.00 & & 1.00 & \\
\hline Married & $1.52(1.35-1.71)$ & $<0.001$ & $1.09(0.90-1.31)$ & 0.413 \\
\hline Widow/widower/divorcee & $2.26(1.88-2.72)$ & $<0.001$ & $1.00(0.76-1.32)$ & 0.992 \\
\hline \multicolumn{5}{|l|}{ Educational level } \\
\hline No formal education & $2.15(1.77-2.62)$ & $<0.001$ & $1.07(0.81-1.40)$ & 0.647 \\
\hline Primary education & $1.54(1.33-1.79)$ & $<0.001$ & $1.13(0.93-1.36)$ & 0.220 \\
\hline Secondary education & $1.08(0.95-1.24)$ & 0.243 & $1.08(0.93-1.26)$ & 0.296 \\
\hline Tertiary education & 1.00 & & 1.00 & \\
\hline \multicolumn{5}{|l|}{ Current work status } \\
\hline Working & 1.00 & & 1.00 & \\
\hline Not working & $1.30(1.17-1.43)$ & $<0.001$ & $0.94(0.82-1.07)$ & 0.337 \\
\hline \multicolumn{5}{|c|}{ Monthly household income (1 MYR $\approx 0.29$ USD) } \\
\hline$<$ MYR1000 & $1.10(0.95-1.26)$ & 0.251 & - & - \\
\hline MYR1000-MYR1999 & $1.07(0.94-1.23)$ & 0.305 & - & - \\
\hline MYR2000-MYR2999 & $1.02(0.88-1.18)$ & 0.822 & - & - \\
\hline$\geq$ MYR3000 & 1.00 & & - & - \\
\hline \multicolumn{5}{|l|}{ LIFESTYLE FACTORS } \\
\hline \multicolumn{5}{|l|}{ BMI status $\left(\mathrm{kg} / \mathrm{m}^{2}\right)$} \\
\hline Underweight $(<18.5)$ & $1.25(1.03-1.51)$ & 0.022 & $1.29(1.05-1.57)$ & 0.014 \\
\hline Normal (18.5-24.9) & 1.00 & & 1.00 & \\
\hline Overweight (25.0-29.9) & $1.05(0.93-1.19)$ & 0.417 & $0.94(0.83-1.07)$ & 0.356 \\
\hline Obese $(\geq 30)$ & $1.25(1.09-1.43)$ & 0.002 & $1.06(0.91-1.23)$ & 0.470 \\
\hline
\end{tabular}


Table 3 Univariate and multivariate logistic regression analyses for poor self-rated health, Malaysian adults aged 18 years and older, by socio-demographic characteristics, lifestyle factors, and chronic disease status (Continued)

\begin{tabular}{|c|c|c|c|c|}
\hline \multicolumn{5}{|c|}{ Consumption of fruits and vegetables } \\
\hline$<5$ servings/day & $1.13(0.92-1.38)$ & 0.235 & $1.10(0.88-1.37)$ & 0.412 \\
\hline$\geq 5$ servings/day & 1.00 & & 1.00 & \\
\hline \multicolumn{5}{|c|}{ Physical activity status } \\
\hline Active & 1.00 & & 1.00 & \\
\hline Inactive & $1.48(1.34-1.63)$ & $<0.001$ & $1.25(1.11-1.39)$ & $<0.001$ \\
\hline \multicolumn{5}{|l|}{ Smoking status } \\
\hline Never smoker & 1.00 & & 1.00 & \\
\hline Former smoker & $1.55(1.31-1.84)$ & $<0.001$ & $1.38(1.12-1.70)$ & 0.003 \\
\hline Current smoker & $0.89(0.79-1.00)$ & 0.047 & $1.08(0.91-1.29)$ & 0.356 \\
\hline \multicolumn{5}{|l|}{ Drinking status } \\
\hline Non-drinker & 1.00 & & 1.00 & \\
\hline Former drinker & $1.15(0.92-1.43)$ & 0.218 & $1.27(1.01-1.62)$ & 0.049 \\
\hline Current drinker & $1.00(0.83-1.20)$ & 0.975 & $1.35(1.08-1.68)$ & 0.008 \\
\hline \multicolumn{5}{|c|}{ CHRONIC DISEASE STATUS } \\
\hline \multicolumn{5}{|l|}{ Asthma } \\
\hline Yes & $1.89(1.60-2.25)$ & $<0.001$ & $1.66(1.36-2.03)$ & $<0.001$ \\
\hline No & 1.00 & & 1.00 & \\
\hline \multicolumn{5}{|l|}{ Arthritis } \\
\hline Yes & $2.60(2.19-3.09)$ & $<0.001$ & $1.87(1.52-2.29)$ & $<0.001$ \\
\hline No & 1.00 & & 1.00 & \\
\hline \multicolumn{5}{|l|}{ Diabetes mellitus } \\
\hline Yes & $1.51(1.29-1.78)$ & $<0.001$ & $0.89(0.72-1.09)$ & 0.261 \\
\hline No & 1.00 & & 1.00 & \\
\hline \multicolumn{5}{|l|}{ Hypertension } \\
\hline Yes & $1.97(1.77-2.20)$ & $<0.001$ & $1.39(1.18-1.64)$ & $<0.001$ \\
\hline No & 1.00 & & 1.00 & \\
\hline \multicolumn{5}{|c|}{ Hypercholesterolemia } \\
\hline Yes & $1.95(1.69-2.26)$ & $<0.001$ & $1.43(1.18-1.74)$ & $<0.001$ \\
\hline No & 1.00 & & 1.00 & \\
\hline \multicolumn{5}{|l|}{ Heart disease } \\
\hline Yes & $2.94(2.38-3.63)$ & $<0.001$ & $1.85(1.43-2.39)$ & $<0.001$ \\
\hline No & 1.00 & & 1.00 & \\
\hline
\end{tabular}

Odds ratios (ORs) adjusted for all other variables shown in the table.

and current drinkers were significantly associated with poor SRH. Previous studies have reported that obesity and underweight are significantly associated with poor SRH [13, 32, 33]; however, our study found that only underweight was significantly associated with poor SRH. This finding was in line with the result of a study in Singapore [34] that found no significant association between obesity and poor SRH. Conflicting findings have emerged regarding the association between BMI and SRH across different studies and populations. Two studies in Greece showed inconsistent findings between urban and rural populations, with obesity associated with poor SRH in urban populations [35] but no significant association between BMI and SRH in rural populations [36]. Another Greek study reported that obesity is associated with good SRH in centenarians [37]. The inconsistent association between BMI and SRH is partially related to differences in perceptions about the body and health in the study populations [38]. In our study, a possible explanation for the association between underweight and poor SRH among Malaysians is that people may be influenced by traditional ideas that being "heavy" 
(overweight/obesity) is "healthy," while being underweight is not healthy [39].

Previous studies have consistently shown that physical activity and exercise have positive effects on SRH [40-43]. Our results generally support this finding by showing that individuals who were physically inactive were more likely to report poor SRH. It is consistent with previous findings from Malaysia reported by Cheah [16,] and also findings from Singapore reported by Ramkumar et al. [34]. The latter studied 409 Singaporean adults aged 40 years and above and found that lack of exercise was associated with poor SRH. Inconsistent findings were reported in a study conducted in Poland that found no positive association between physical activity and SRH [44]. The contradictory findings may be related to the Poland study [44] measuring physical activity only in terms of leisure time while our study measured total physical activity including different forms of physical activity (e.g., work-related, transportation, leisure-time, and domestic/gardening). A recent study of physical activity using the same national survey data (NHMS 2011) reported that about two in five Malaysian adults were physically inactive, particularly women and the elderly [45]. In the current study, these two groups were more likely to report poor SRH.

In contrast to findings from the 2007 Behavioural Risk Factor Surveillance System (BRFSS) survey conducted in the United States [46] and the Wellbeing, Eating and Exercise for a Long Life (WELL) study in Australia [47], our study did not show any relationship between consumption of fruits and vegetables and SRH. However, it should be noted that the survey methods, the questions used to measure consumption of fruits and vegetables, and the response options in the two studies were different from those used in our study. It is also possible that the measurement of servings of fruits and vegetables consumed per day was inaccurate because of selfreporting, which is subject to recall bias. However, our results were in line with studies by Darviri et al. [35] and Kwasniewska et al. [44], which reported no significant association between consumption of fruits and vegetables and SRH. The association between consumption of fruits and vegetables and SRH would benefit from further investigation using a more detailed questionnaire.

In our study, no statistically significant difference was found in poor SRH for current smokers compared with never smokers. This finding is contrary to previous studies $[12,13,48]$, that reported a significant association between current smoking and poor SRH. A possible explanation is that smoking is socially acceptable and is considered a norm in Malaysian culture and society, particularly among men [49]; thus, the relationship between smoking and poor SRH may be confounded. Studies in Greece [35] and Japan [50] have consistently found a strong association between current heavy smokers ( $>20$ cigarettes/day) and poor SRH, while no significant association was found for current light smokers $(\leq 20$ cigarettes/day). This may be because heavy smokers are more likely than light smokers to perceive themselves at higher risk of developing smoking-related diseases such as lung cancer or chronic lung disease [51]. Our results showed that former smokers had significantly increased odds of poor SRH compared with never smokers. Former smokers reported higher odds of poor $\mathrm{SRH}$, possibly because of personal health problems that led them to quit smoking [52]. Further study is needed to explore the relations between former smokers and SRH.

In line with previous studies $[46,53,54]$ that support the negative effects of alcohol consumption on SRH, our study showed that current drinkers were associated with greater likelihood of poor SRH compared with non-drinkers. However, our results contradict those obtained in Spain [55], which reported better health status among alcohol drinkers than non-drinkers. In Spain, the habit of alcohol consumption has developed within a context of social and leisure activities that contribute to a more positive SRH [56]. The different cultural contexts between Western and Asian countries may account for these differences in SRH. Interestingly, a study in China found a significant association between alcohol drinkers and good SRH but the authors suggested that this finding may have been caused by lack of specific information about the drinking habits of respondents, particularly the number of units consumed and the frequency of alcohol consumption, in the analyses [57]. Results on lifestyle factors vary broadly across studies and populations. In general, individuals who adopt a more proactive lifestyle report better health ratings than those who do not [33, 36, 44, 46, 47].

There is substantial evidence supporting the negative impact of chronic diseases on SRH $[3,16,34,58]$. People with chronic diseases can experience pain and disability that results in poor SRH. Our study showed that chronic diseases such as asthma, arthritis, hypertension, hypercholesterolemia, and heart disease were strongly associated with poor SRH, as reported in the literature. However, we did not find a significant association between diabetes mellitus and poor SRH. A possible explanation for this is that people with diabetes may also have been diagnosed with hypertension and/ or hypercholesterolemia. The presence of these chronic diseases may attenuate the effects of diabetes on SRH, resulting in a finding of non-significance. It is also possible that the respondents in our study who had disbetes mostly seek care from public health clinics that have effective diabetes control programmes and diabetes nurse educator services, resulting in these people having better self-care practices for diabetes management. The 
findings on diabetes and SRH indicate a need for further investigation.

While this study emphasised the associations between lifestyle, chronic diseases, and SRH, several sociodemographic factors such as age, sex, ethnicity, and residence (urban/rural) were shown to have statistically significant influences on SRH in Malaysia. Older people were more likely to report poor SRH than younger people, which has been shown in other studies $[12,13,35]$. Women were more likely to report poor SRH than men. This finding is related to women being more likely than men to report in their general health assessment a wide range of health-related problems from simple illness or chronic diseases as well as non-health-related problems such as anxiety, depression, or a recent negative life event $[59,60]$. Ethnic differences in SRH may be due to cultural differences in how individuals perceive their health status. Other Bumiputeras reported the highest prevalence of poor $\mathrm{SRH}$, compared with other ethnic groups, probably because the majority live in rural areas. People in rural areas were more likely than those in urban areas to rate their health status as poor; this finding may be related to limited accessibility to health care services and lack of financial means to pay for services.

The major strengths of this study are the representativeness and large sample size. The results can be extrapolated accurately and generalised to the Malaysian adult population. Furthermore, the participants were interviewed face-to-face by trained interviewers and the response rate was higher than reported for other studies. However, the study has several limitations that should be noted. The main limitation is the cross-sectional design of the study, which does not allow for determination of causal effect relationships. Another limitation is the possibility of recall bias associated with self-reported data on smoking, alcohol consumption, physical activity, and consumption of fruits and vegetables. Third, the "social desirability" of certain answer categories is a concern in face-to-face interviews. To project a favourable image, respondents may give answers that are deemed more "socially accepted" than their "true" answers. Finally, several other factors found to be associated with SRH in previous studies (e.g., psychosocial and environmental factors) were not investigated in this study.

\section{Conclusions}

The prevalence of poor SRH among Malaysian adults is $20.1 \%$, which increases with age. Several negative lifestyle behaviours and chronic diseases were significantly associated with poor SRH among Malaysian adults. Additional public health strategies are needed to promote healthy lifestyles and disease prevention interventions should be implemented to improve personal and community health. More preventive measures and health care services targeting chronic diseases are urgently needed, particularly in rural communities. Further research that takes into account the influences of other potential confounders such as psychososial and environmental factors is needed to fully understand the correlates and determinants of SRH.

\section{Additional file}

\begin{abstract}
Additional file 1: Table S1. Percent distribution of self-rated health status (five SRH cetegories), Malaysian adults aged 18 years and older, NHMS 2011 (DOCX $15 \mathrm{~kb}$ )
\end{abstract}

\section{Competing interests}

The authors declare that they have no competing interests.

\section{Authors' contributions}

CYY performed the statistical analyses, interpreted the findings, and drafted the manuscript. TCH, LKK, LKH and YPS contributed to the interpretation of results and preparation of the manuscript. KCC participated in the interpretation of results and revised the manuscript. MAO and NAA were involved in the design and coordination of the study and the revision of the manuscript. All authors read and approved the final manuscript.

\section{Acknowledgements}

The authors would like to thank the Director General of Health, Malaysia for granting permission to publish this study. We also thank those who were involved in the study and assisted with data collection and management for their contributions and commitment. We are grateful to all the study participants for their kind cooperation. This survey was funded by the Ministry of Health, Malaysia (NMRR-10-757-6837).

\section{Author details}

${ }^{1}$ Institute for Public Health, National Institutes of Health, Ministry of Health Malaysia, Kuala Lumpur, Malaysia. ${ }^{2}$ Institute for Medical Research, National Institutes of Health, Ministry of Health Malaysia, Kuala Lumpur, Malaysia.

Received: 16 December 2014 Accepted: 22 July 2015

Published online: 06 August 2015

\section{References}

1. World Health Organization. Global health risks: Mortality and burden of disease attributable to selected major risks. Geneva: WHO; 2009.

2. Mora RR. Lifestyle medicine: the importance of considering all the causes of disease. Rev Psiquiatr Salud Ment. 2012;5:48-52.

3. Molarius A, Janson S. Self-rated health, chronic diseases, and symptoms among middle-aged and elderly men and women. J Clin Epidemiol. 2002;55:364-70.

4. Pisinger C, Toft U, Aadahl M, Glumer C, Jorgensen T. The relationship between lifestyle and self-reported health in a general population The Inter99 study. Prev Med. 2009;49:418-23.

5. Harrington J, Perry IJ, Lutomski J, Fitzgerald AP, Shiely F, McGee H, et al. Living longer and feeling better: healthy lifestyle, self-rated health, obesity and depression in Ireland. Eur J Public Health. 2010;20:91-5.

6. Cummins S, Stafford M, Macintyre S, Marmot M, Ellaway A. Neighbourhood environment and its association with self rated health: evidence from Scotland and England. J Epidemiol Community Health. 2005;59:207-13.

7. Poortinga W, Dunstan FD, Fone DL. Neighbourhood deprivation and selfrated health: The role of perceptions of the neighbourhood and of housing problems. Health Place. 2008;14:562-75.

8. Freidoony L, Chhabi R, Kim CS, Park MB, Kim CB. The components of selfperceived health in the Kailali district of Nepal: A cross-sectional survey. Int J Environ Res Public Health. 2015;12:3215-31.

9. DeSalvo KB, Bloser N, Reynolds K, He J, Muntner P. Mortality prediction with a single general self-rated health question, A meta-analysis. J Gen Intern Med. 2006;21:267-75. 
10. Bombak AE. Self-rated health and public health: a critical perspective. Front Public Health. 2013;1:15-8. doi:10.3389/fpubh.2013.00015.

11. Jylha M. What is self-rated health and why does it predict mortality? Towards a unified conceptual model. Soc Sci Med. 2009;69:307-16.

12. Lim WY, Ma S, Heng D, Bhalla V, Chew SK. Gender, ethnicity, health behaviour \& self-rated health in Singapore. BMC Public Health. 2007;7:184.

13. Ahmad K, Jafar TH, Chaturvedi N. Self-rated health in Pakistan: results of a national health survey. BMC Public Health. 2005:5:51.

14. Wang N, Iwasaki M, Otani T, Hayashi R, Miyazaki H, Xiao L, et al. Perceived health as related to income, socio-economic status, lifestyle, and social support factors in a middle-aged Japanese. J Epidemiol. 2005;15:155-62.

15. Haseen F, Adhikari R, Soonthorndhada K. Self-assessed health among Thai elderly. BMC Geriatr. 2010;10:30.

16. Cheah YK. An exploratory study on self-rated health status: The case of Penang, Malaysia. Malays J Econ Studies. 2012;49:141-55.

17. Haron SA, Sharpe DL, Masud J, Abdel-Ghany M. Health divide: Economic and demographic factors associated with self-rated health among older Malaysians. J Fam Econ Issues. 2010;31:328-37.

18. Ng ST, Tengku-Aizan H, Tey NP. Perceived health status and daily activity participation of older Malaysians. Asia Pac J Public Health. 2011;23:470-84.

19. Fadhli Y, Azahadi O, Noor Ani A, Balkish MN, Ahmad Jessree K, Tahir A. Approaches in methodology of a population-based study in Malaysia: The National Health and Morbidity Survey 2011 (NHMS 2011). Malays J Med Health Sci. 2013;9:25-33. ISSN 1675-8544.

20. World Health Organization. Obesity: Preventing and managing the global epidemic. Report of a WHO consultation. WHO Technical Report Series 894. Geneva: WHO; 2000.

21. World Health Organization. STEPwise approach to noncommunicable disease risk factor surveillance (STEPS). http://www.who.int/chp/steps/ riskfactor/en. Accessed June 29, 2015.

22. World Health Organization. Diet, nutrition and the prevention of chronic diseases. Report of a joint WHO/FAO expert consultation on diet nutrition and the prevention of chronic diseases. Geneva: WHO; 2003.

23. Craig CL, Marshall AL, Sjostrom M, Bauman AE, Booth ML, Ainsworth BE, et al. International physical activity questionnaire: 12-country reliability and validity. Med Sci Sports Exerc. 2003;35:1381-95.

24. Guidelines for data processing and analysis of the International Physical Activity Questionnaire (IPAQ). 2005. https://sites.google.com/site/theipaq/ scoring-protocol. Accessed September 27, 2014.

25. Bursac Z, Gauss CH, Williams DK, Hosmer DW. Purposeful selection of variables in logistic regression. Source Code Biol Med. 2008;3:17.

26. Shibuya $\mathrm{K}$, Hashimoto $\mathrm{H}$, Yano E. Individual income, income distribution, and self rated health in Japan: cross sectional analysis of nationally representative sample. BMJ. 2002;324:16-9.

27. Health Canada. A statistical profile on the health of first nations in Canada: Self-rated health and selected conditions, 2002 to 2005. http://www.hcsc.gc.ca/fniah-spnia/pubs/aborig-autoch/index-eng.php. Accessed October $18,2014$.

28. Centers for Disease Control and Prevention (CDC). Racial/ethnic disparities in self-rated health status among adults with and without disabilities - United States, 2004-2006. MMWR Morb Mortal Wkly Rep. 2008;57:1069-73.

29. Jurges $\mathrm{H}$, Avendano M, Mackenbach JP. Are different measures of self-rated health comparable? An assessment in five European countries. Eur J Epidemiol. 2008;23:773-81.

30. Hanibuchi T, Nakaya T, Murata C. Socio-economic status and self-rated health in East Asia: a comparison of China, Japan, South Korea and Taiwan. Eur J Public Health. 2012;22:47-52.

31. Zavras D, Tsiantou V, Pavi E, Mylona K, Kyriopoulos J. Impact of economic crisis and other demographic and socio-economic factors on self-rated health in Greece. Eur J Public Health. 2013;23:206-10.

32. Ford ES, Moriarty DG, Zack MM, Mokdad AH, Chapman DP. Self-reported body mass index and health-related quality of life: findings from the Behavioral Risk Factor Surveillance System. Obes Res. 2001;9:21-31.

33. Molarius A, Berglund K, Eriksson C, Lambe M, Nordstrom E, Eriksson HG, et al. Socioeconomic conditions, lifestyle factors, and self-rated health among men and women in Sweden. Eur J Public Health. 2007;17:125-33.

34. Ramkumar A, Quah JL, Wong T, Yeo LS, Nieh CC, Shankar A, et al. Self-rated health, associated factors and diseases: a community-based cross-sectional study of Singaporean adults aged 40 years and above. Ann Acad Med Singapore. 2009;38:606-7.
35. Darviri C, Artemiadis AK, Tigani X, Alexopoulos EC. Lifestyle and selfrated health: a cross-sectional study of 3,601 citizens of Athens. Greece BMC Public Health. 2011;11:619.

36. Darviri C, Fouka G, Gnardellis C, Artemiadis AK, Tigani X, Alexopoulos EC. Determinants of self-rated health in a representative sample of a rural population: a cross-sectional study in Greece. Int J Environ Res Public Health. 2012;9:943-54.

37. Tigani $X$, Artemiadis AK, Alexopoulos EC, Chrousos GP, Darviri C. Self-rated health in centenarians: a nation-wide cross-sectional Greek study. Arch Gerontol Geriatr. 2012;54:e342-8.

38. Imai K, Gregg EW, Chen YJ, Zhang P, de Rekeneire N, Williamson DF. The association of BMI with functional status and self-rated health in US adults. Obesity (Silver Spring). 2008;16:402-8.

39. Pu C, Chou YJ. Health ratings for underweight, overweight and obese adolescents: disparities between adolescent's own report and the parent's report. Asia Pac J Clin Nutr. 2010;19:180-7.

40. Brown DW, Balluz LS, Heath GW, Moriarty DG, Ford ES, Giles WH, et al. Associations between recommended levels of physical activity and health-related quality of life. Findings from the 2001 Behavioral Risk Factor Surveillance System (BRFSS) survey. Prev Med. 2003;37:520-8.

41. Sodergren M, Sundquist J, Johansson SE, Sundquist K. Physical activity, exercise and self-rated health: a population-based study from Sweden. BMC Public Health. 2008:8:352.

42. Han MA, Kim KS, Park J, Kang MG, Ryu SY. Association between levels of physical activity and poor self-rated health in Korean adults: The Third Korea National Health and Nutrition Examination Survey (KNHANES), 2005. Public Health. 2009;123:665-9.

43. Rosenkranz RR, Duncan MJ, Rosenkranz SK, Kolt GS. Active lifestyles related to excellent self-rated health and quality of life: cross sectional findings from 194,545 participants in the 45 and up study. BMC Public Health. 2013;13:1071.

44. Kwasniewska M, Kaleta D, Dziankowska-Zaborszczyk E, Drygas W, Makowiec-Dabrowska T. Lifestyle index and self-rated health status. Int J Occup Med Environ Health. 2007;20:349-56

45. Teh CH, Lim KK, Chan YY, Lim KH, Azahadi O, Hamizatul Akmar AH, et al. The prevalence of physical activity and its associated factors among Malaysian adults: findings from the National Health and Morbidity Survey 2011. Public Health. 2014;128:416-23.

46. Tsai J, Ford ES, Li C, Zhao G, Pearson WS, Balluz LS. Multiple healthy behaviors and optimal self-rated health: findings from the 2007 Behavioral Risk Factor Surveillance System Survey. Prev Med. 2010;51:268-74.

47. Sodergren M, McNaughton SA, Salmon J, Ball K, Crawford DA. Associations between fruit and vegetable intake, leisure-time physical activity, sitting time and self-rated health among older adults: crosssectional data from the WELL study. BMC Public Health. 2012;12:551.

48. Wang MP, Ho SY, Lo WS, Lai MK, Lam TH. Smoking is associated with poor selfrated health among adolescents in Hong Kong. Nicotine Tob Res. 2012;14:682-7.

49. Robson N, Bond A, Wolff K. A comparison of smoking behaviour characteristics between Caucasian smokers in the United Kingdom and Malay smokers in Malaysia. Prev Med. 2013;57(Suppl):S8-S10.

50. Nakata A, Takahashi M, Swanson NG, Ikeda T, Hojou M. Active cigarette smoking, secondhand smoke exposure at work and home, and self-rated health. Public Health. 2009;123:650-6.

51. Hayes RB, Borrelli B. Differences between Latino daily light and heavier smokers in smoking attitudes, risk perceptions, and smoking cessation outcome. Nicotine Tob Res. 2013;15:103-11.

52. Nakamura K, Sakurai M, Nishijo M, Morikawa Y, Nakagawa H. Characteristics of smoking cessation in former smokers in a rural area in Japan. Int J Prev Med. 2012;3:459-65.

53. Okosun IS, Seale JP, Daniel JB, Eriksen MP. Poor health is associated with episodic heavy alcohol use: evidence from a National Survey. Public Health. 2005;119:509-17.

54. Tsai J, Ford ES, Li C, Pearson WS, Zhao G. Binge drinking and suboptimal self-rated health among adult drinkers. Alcohol Clin Exp Res. 2010;34:1465-71.

55. Valencia-Martin JL, Galan I, Guallar-Castillon P, Rodriguez-Artalejo F. Alcohol drinking patterns and health-related quality of life reported in the Spanish adult population. Prev Med. 2013;57:703-7.

56. Giron P. Determinants of self-rated health in Spain: differences by age groups for adults. Eur J Public Health. 2012;22:36-40.

57. Haseli-Mashhadi N, Pan A, Ye X, Wang J, Qi Q, Liu Y, et al. Self-Rated Health in middle-aged and elderly Chinese: distribution, determinants and 
associations with cardio-metabolic risk factors. BMC Public Health. 2009;9:368.

58. Li C, Ford ES, Zhao G, Mokdad AH. Associations of health risk factors and chronic illnesses with life dissatisfaction among U.S. adults: the Behavioral Risk Factor Surveillance System, 2006. Prev Med. 2009:49:253-9.

59. Singh L, Arokiasamy P, Singh PK, Rai RK. Determinants of gender differences in self-rated health among older population: evidence from India. SAGE Open. 2013:3:1-2. doi:10.1177/2158244013487914.

60. Benyamini $Y$, Leventhal EA, Leventhal $\mathrm{H}$. Gender differences in processing information for making self-assessments of health. Psychosom Med. 2000;62:354-64

\section{Submit your next manuscript to BioMed Central} and take full advantage of:

- Convenient online submission

- Thorough peer review

- No space constraints or color figure charges

- Immediate publication on acceptance

- Inclusion in PubMed, CAS, Scopus and Google Scholar

- Research which is freely available for redistribution 